\title{
Pulmonary artery sling: definitive diagnosis by colour Doppler flow mapping avoiding cardiac catheterisation
}

\author{
J P Gnanapragasam, A B Houston, M P G Jamieson
}

\begin{abstract}
Pulmonary artery sling is a rare abnormality that usually presents in infancy with wheeze or stridor. Diagnoses by cross sectional echocardiography, computed tomography, and magnetic resonance imaging have been described but pulmonary arteriography was regarded as essential for a definite diagnosis. The use of colour Doppler adds to the diagnostic certainty of cross sectional echocardiography and colour Doppler provided a definite diagnosis in an infant that allowed surgical repair without the need for cardiac catheterisation. Postoperative flow through the left pulmonary artery was also assessed by Doppler, and this avoided the need for repeat catheterisation or perfusion lung scanning.
\end{abstract}

An aberrant left pulmonary artery or pulmonary artery sling usually presents in early infancy with signs of respiratory obstruction. In about $40 \%$ of cases it is associated with other cardiac abnormalities ${ }^{1}$ but cardiac symptoms or signs are often not evident at presentation. The abnormality cannot be diagnosed clinically and the diagnosis rests on investigations.

The aberrant left pulmonary artery may be shown by cross sectional echocardiography, ${ }^{2}$ computed tomography, ${ }^{3}$ and magnetic resonance imaging. " None the less, pulmonary arteriography has been considered essential for confirmation of the diagnosis..$^{3-5}$ We report a patient in whom the diagnosis was confidently established by colour Doppler combined with cross sectional echocardiography and surgical repair was undertaken without cardiac catheterisation.

\section{Case report}

A 3 month old boy presented with intermittent wheeze and stridor. Clinical examination and $x$ ray of the chest were normal. Barium swallow examination did not show any abnormalities. In view of the stridor, bronchoscopy was carried out and showed a posterior pulsatile indentation in the trachea that suggested a pulmonary sling. The infant was referred for a cardiac assessment at this stage and an echocardiogram was performed. Parasternal short axis and subxiphoid views showed that the main pulmonary artery did not bifurcate in the usual manner and showed a break in the continuity of the posterior wall of the right pulmonary artery with an echo free space leading from it (fig 1). Colour Doppler confirmed that the echo free space was an aberrant artery and not an artefact because it showed flow into the space from the right pulmonary artery (fig 2). The aortic arch was normal and there were no intracardiac abnormalities. On the basis of these findings, surgical repair was carried out with subsequent resolution of the respiratory symptoms. Colour Doppler flow mapping performed three months after operation showed unobstructed flow into the left pulmonary artery. The maximum velocity across the origin of the left pulmonary artery was only $1.9 \mathrm{~m} / \mathrm{s}$, confirming that there was no stenosis.

\section{Discussion}

Pulmonary artery sling is a rare cause of respiratory obstruction in early infancy. A barium swallow examination is often the initial diagnostic investigation carried out in infants presenting with significant stridor or wheeze and the classic anterior indentation of the oesophagus produced by the aberrant left pulmonary artery may be seen. On the other hand, the barium swallow may be normal in more than $20^{\circ}{ }_{0}$ of cases ${ }^{1}$ and an anterior indentation may also be caused by a bronchial cyst, reduplication of the oesophagus, enlarged lymph nodes, and a rare form of aberrant right subclavian artery. Thus barium swallow examination cannot be relied upon as a definitive diagnostic test.

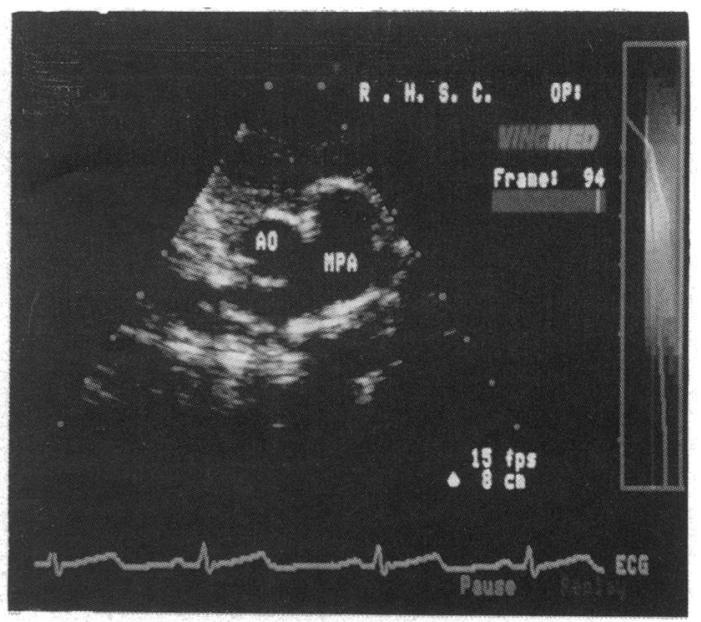

Figure 1 Parasternal short axis view showing absence of normal bifurcation of the main pulmonary artery. The aberrant origin of the left pulmonary artery is not conclusively shown. $A O$, aorta; $M P A$, main pulmonary artery. 
Figure 2 Same frame as fig 1 but with colour Doppler flow mapping showing flow from the righ pulmonary artery into the aberrant vessel which courses posteriorly and leftwards. $A O$, aorta; $M P A$, main pulmonary artery.

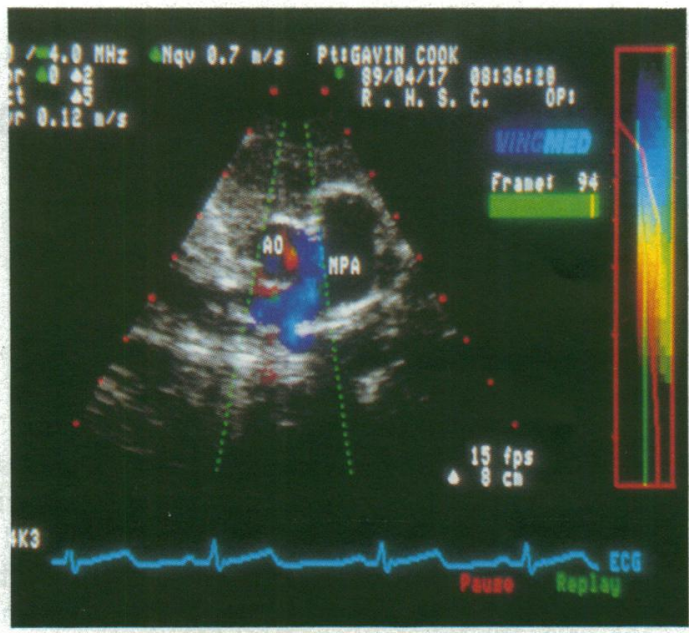

Magnetic resonance imaging ${ }^{4}$ and computed tomography ${ }^{3}$ have been proposed as screening tests for a pulmonary sling but these techniques are not readily available, they require the infant to be motionless, which necessitates sedation, and further investigations are considered necessary to confirm the diagnosis and rule out associated cardiac malformations. Cross sectional echocardiographic findings in pulmonary artery sling were described by Yeager et al who commented that the anomalous artery may not be apparent on imaging in the presence of hyperinflation of the lungs, a finding that is not uncommon in this condition. ${ }^{2}$ They suggested that cross sectional echocardiography was a useful screening test but believed that pulmonary arteriography was a more specific and sensitive investigation. In cases of pulmonary artery sling, bifurcation of the main pulmonary artery is not demonstrable on imaging and the aberrant vessel may be seen to arise from the proximal right pulmonary artery and run posteriorly and leftwards. Owing to the depth of imaging and overlapping of the lungs there may be some uncertainty as to whether the echo free space seen is an aberrant left pulmonary artery or an artefact caused by echo drop out. Since the initial posterior course of the anomalous pulmonary artery is in line with the Doppler transducer in the parasternal and subxiphoid short axis views, blood flow into it from the right pulmonary artery should be readily demonstrated by colour Doppler flow mapping. The aberrant origin of the left pulmonary artery from the proximal right pulmonary artery can thus be firmly established, avoiding the need for invasive cardiac catheterisation.

In view of the high incidence of postoperative occlusion of the left pulmonary artery it has been emphasised that follow up cardiac catheterisation is essential in all cases to evaluate flow through the left pulmonary artery. ${ }^{6}$ This is no longer necessary because colour Doppler flow mapping provides an excellent means of assessing flow through the left pulmonary artery. If occlusion is present, reoperation is necessary to prevent the possible development of pulmonary hypertension.

Although a pulmonary sling or a vascular ring is an uncommon cause of stridor in infancy it is an important factor to consider because the infant may rapidly deteriorate and die before the diagnosis is made. Thus echocardiography with colour Doppler should be performed early in the assessment of all infants with appreciable stridor as it may show a vascular ring ${ }^{7}$ or a pulmonary sling

J P G is supported by a grant from the Scottish Home and Health Department.

1 Gikony^ BM, Jue KL, Edwards JE. Pulmonary vascula sling: report of seven cases and review of the literature. Pediatr Cardiol 1989;10:81-9.

2 Yeager SB, Chin AJ, Sanders SP. Two-dimensional echocardiographic diagnosis of pulmonary artery sling in echocardiographic diagnosis of pulmonary

3 Rheuban KS, Ayres N, Still JG, Alford B. Pulmonary artery sling: a new diagnostic tool and clinical review. Pediatrics

4 Malmgren N, Laurin S, Lundstrom NR. Pulmonary artery sling. Diagnosis by magnetic resonance imaging. Acta Radiol 1987;29:7-9.

5 Park SC, Zuberbuhler JR. Vascular ring and pulmonary sling. In: Anderson RH, Macartney FJ, Shinebourne EA Tynan M, eds. Paediatric cardiology. Edinburgh: Churchill Livingstone, 1987:1133-5.

6 Lenox CC, Crisler C, Zuberbuhler JR, et al. Anomalous left pulmonary artery. Successful management. J Thorac Cardiovasc Surg 1979;77:748-52.

7 Kan M-N, Nanda NC, Stopa AR. Diagnosis of double aortic arch by cross sectional echocardiography with Dopple colour flow mapping. Br Heart $J$ 1987;58:284-6. 